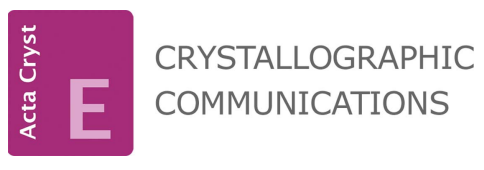

ISSN 2056-9890

Received 15 September 2020

Accepted 29 September 2020

Edited by W. T. A. Harrison, University of Aberdeen, Scotland

Keywords: $\mathrm{TIMgCl}_{3}$; scintillator; crystal structure; high-temperature neutron diffraction.

CCDC reference: 2034695

Supporting information: this article has supporting information at journals.iucr.org/e

\section{The crystal structure of $\mathrm{TIMgCl}_{3}$ from $290 \mathrm{~K}$ to $725 \mathrm{~K}$}

\author{
Drew R. Onken, ${ }^{a *}$ Didier Perrodin, ${ }^{a}$ Sven C. Vogel, ${ }^{b}$ Edith D. Bourret ${ }^{\mathrm{a}}$ and Federico \\ Moretti $^{\mathrm{a}}$
}

aLawrence Berkeley National Laboratory, Berkeley, CA 94720, USA, and ${ }^{\mathbf{b}}$ Los Alamos National Laboratory, Los Alamos,
NM 87545, USA. *Correspondence e-mail: DOnken@lbl.gov

The title compound, thallium magnesium trichloride, has been identified as a scintillator with both moderate gamma-stopping power and moderate light yield. Knowledge of its crystal structure is needed for further development. This work determines the crystal structure of $\mathrm{TlMgCl}_{3}$ to be hexagonal $P 6_{3} / m m c$ (No. 194) and isostructural with $\mathrm{RbMgCl}_{3}$, contrary to previously reported data. This structure was obtained by single-crystal X-ray diffraction and was further confirmed by neutron diffraction measurements. Extending neutron diffraction measurements to high temperature, the data show that $\mathrm{TlMgCl}_{3}$ maintains this crystal structure from $290 \mathrm{~K}$ up through $725 \mathrm{~K}$, approaching the melting point of $770 \mathrm{~K}$. Anisotropic thermal expansion coefficients increase over this temperature range, from 31 to $38 \times 10^{-6} \mathrm{~K}^{-1}$ along the $a$ axis and from 19 to $34 \times 10^{-6}$ $\mathrm{K}^{-1}$ along the $c$ axis.

\section{Chemical context}

In the ongoing search for inorganic scintillators with high gamma-stopping power, $\mathrm{TlMgCl}_{3}$ has been identified. As a result of the presence of thallium, $\mathrm{TlMgCl}_{3}$ has a high effective atomic number, $Z_{\text {eff }}=67$ [calculation methodology (Derenzo \& Choong, 2009) in the supporting information], and a moderate density, $\rho=4.47 \mathrm{~g} \mathrm{~cm}^{-3}$ (determined in this work). A pair of initial crystal growths of $\mathrm{TlMgCl}_{3}$ have been conducted to assess the scintillation properties: Fujimoto et al. (2016) measured 46,000 $\mathrm{ph} \mathrm{MeV}^{-1}$ light yield with 5\% energy resolution at $662 \mathrm{keV}$, and Hawrami et al. (2017) measured $30,600 \mathrm{ph} \mathrm{MeV}^{-1}$ light yield with $3.7 \%$ energy resolution at $662 \mathrm{keV}$.

To develop this compound further, a precise determination of the crystal structure is necessary. This will enable firstprinciples calculations of the electronic configuration and may be useful in assessing challenges that arise during synthesis (e.g. from thermal stresses). This work reports the crystal structure of $\mathrm{TlMgCl}_{3}$ between $290 \mathrm{~K}$ and $725 \mathrm{~K}$, approaching the melting point of $770 \mathrm{~K}$. Previous work on $\mathrm{TlMgCl}_{3}$ by Beznosikov (1978) used powder diffraction to report the space group at room temperature as orthorhombic $(a=6.54, b=$ $9.22, c=6.99 \AA$ ). However, despite using the same synthesis procedure, the structure reported by Beznosikov does not fit the diffraction data reported herein. Arai et al. (2020) published diffraction data but did not provide information on the crystal structure. 
Table 1

Selected geometric parameters $\left(\AA,^{\circ}\right)$.

\begin{tabular}{llll}
\hline $\mathrm{T} 11-\mathrm{Cl} 1$ & $3.5126(2)$ & $\mathrm{Tl} 2-\mathrm{Cl} 2^{\mathrm{iv}}$ & $3.622(5)$ \\
$\mathrm{Tl} 1-\mathrm{Cl} 2^{\mathrm{i}}$ & $3.576(5)$ & $\mathrm{Mg} 1-\mathrm{Cl} 2$ & $2.448(6)$ \\
$\mathrm{T} 2-\mathrm{Cl} 1^{\mathrm{ii}}$ & $3.510(3)$ & $\mathrm{Mg} 1-\mathrm{Cl} 1$ & $2.499(6)$ \\
$\mathrm{Tl} 2-\mathrm{Cl} 2^{\mathrm{iii}}$ & $3.5146(3)$ & $\mathrm{Mg} 2-\mathrm{Cl} 2$ & $2.476(4)$ \\
& & & \\
$\mathrm{Mg} 1^{\mathrm{v}}-\mathrm{Cl} 1-\mathrm{Mg} 1$ & $78.5(3)$ & $\mathrm{Mg} 1-\mathrm{Cl} 2-\mathrm{Mg} 2$ & $178.8(3)$ \\
\hline
\end{tabular}

Symmetry codes: (i) $-y+1, x-y, z$; (ii) $-y, x-y, z$; (iii) $x-1, y,-z+\frac{1}{2}$; (iv) $x-y, x, z-\frac{1}{2}$; (v) $x, y,-z+\frac{1}{2}$.

\section{Structural commentary}

Single crystal X-ray diffraction (SC-XRD) determined $\mathrm{TlMgCl}_{3}$ to have a hexagonal structure (space group $P 6_{3} / \mathrm{mmc}$, No. 194) with lattice parameters $a=7.0228(4), c=$ $17.4934(15) \AA$ at 290 K. Fig. 1 visualizes the unit cell, which shows a three-dimensional corner- and face-sharing framework of six-coordinated $\mathrm{Mg}$ atoms encapsulating the 12coordinated $\mathrm{Tl}$ atoms. There are six formula units in the unit cell. There are two thallium, two magnesium and two chlorine atoms in the asymmetric unit of $\mathrm{TlMgCl}_{3}$, with site symmetries of $\overline{6} m 2$ and $3 m ; 3 m$ and $\overline{3} m ; m m 2$ and $m$, respectively; key bond distances and angles are listed in Table 1. Pairs of Mg2centered octahedra share faces (via $3 \times \mathrm{Cl} 1)$ and these octahedral pairs share corners (via $\mathrm{Cl} 2$ ) with the $\mathrm{Mg} 1$ octahedra to generate an $A B A C B C$ hexagonal stacking sequence of the chloride ions in the $c$-axis direction with the thallium cations occupying the vacant 12 -coordinate sites. The coordination polyhedra of the chloride ions are distorted $\mathrm{ClMg}_{2} \mathrm{Tl}_{4}$ octahedra with the $\mathrm{Mg}^{2+}$ ions in a cis disposition for $\mathrm{Cl} 1$ and a trans disposition for $\mathrm{Cl} 2$. The title compound is isostructural with $\mathrm{RbMgCl}_{3}$ as reported by Devaney et al. (1981) and $\mathrm{RbMnCl}_{3}$ as reported by Goodyear et al. (1977), who describe the structure in more detail. This structure is more complex than that of $\mathrm{CsMgCl}_{3}$ (McPherson et al., 1970), which also has space group $\mathrm{P}_{3} / \mathrm{mmc}$ but only requires two formula units per unit cell and has an $A B$ hexagonal stacking sequence of the chloride ions in the $c$-axis direction.

Neutron diffraction (ND) conducted on powder samples produced diffraction patterns that were in agreement with the crystal structure determined by SC-XRD. Neutron diffraction was conducted at temperatures ranging from $300 \mathrm{~K}$ to $725 \mathrm{~K}$.

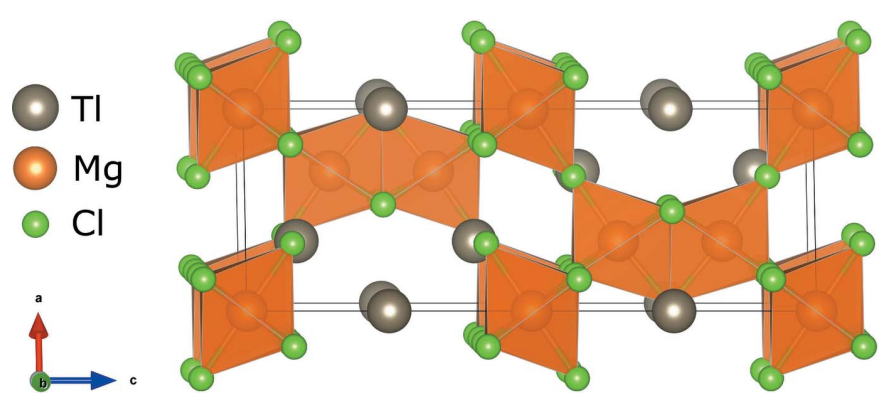

Figure 1

The unit cell of $\mathrm{TlMgCl}_{3}$, with the $\mathrm{MgCl}_{6}$ octahedra shown in polyhedral representation.
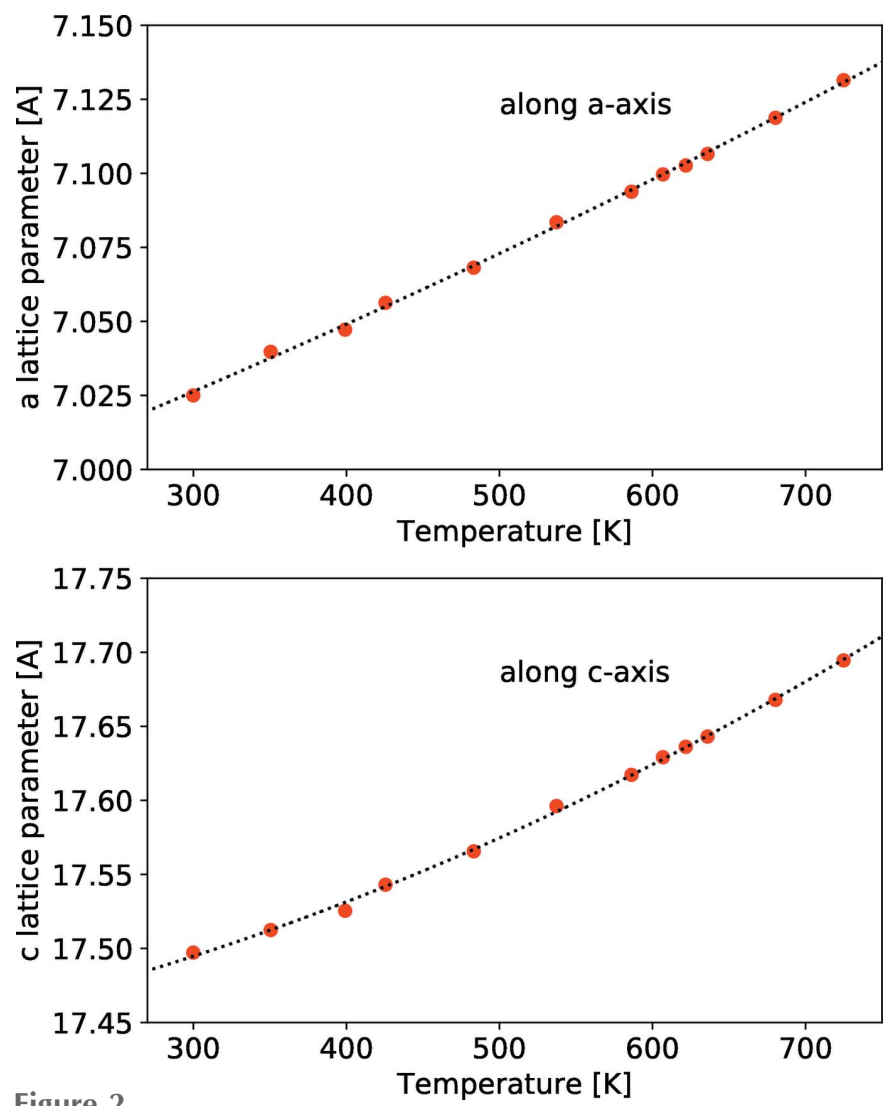

Figure 2

The hexagonal lattice parameters of $\mathrm{TlMgCl}_{3}$ as a function of temperature, from neutron diffraction data. Vertical error bars from Rietveld fitting are within the size of the symbols and are omitted. The dashed lines are second-order polynomial fits to the data.

$\mathrm{TlMgCl}_{3}$ maintains the same $P 6_{3} / m m c$ crystal structure over this measured temperature range (see supporting information for more details on the powder ND data and fits). Fig. 2 shows the lattice parameters as a function of temperature. From these data, the thermal expansion along each axis is calculated

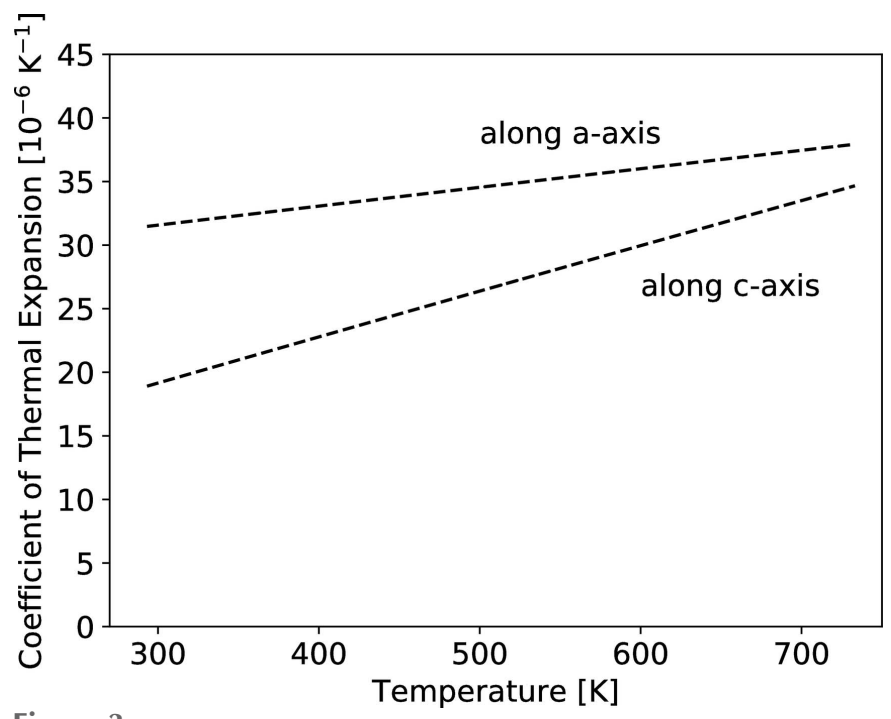

Figure 3

Thermal expansion coefficients as a function of temperature, calculated from the second-order polynomial fit of the lattice parameters in Fig. 2. 
Table 2

Experimental details.

\begin{tabular}{|c|c|}
\hline \multicolumn{2}{|l|}{ Crystal data } \\
\hline Chemical formula & $\mathrm{TlMgCl}_{3}$ \\
\hline$M_{\mathrm{r}}$ & 335.03 \\
\hline Crystal system, space group & Hexagonal, $\mathrm{P6}_{3} / m m c$ \\
\hline Temperature (K) & 290 \\
\hline$a, c(\AA)$ & $7.0228(4), 17.4934(15)$ \\
\hline$V\left(\AA^{3}\right)$ & $747.18(11)$ \\
\hline$Z$ & 6 \\
\hline Radiation type & Mo $K \alpha$ \\
\hline$\mu\left(\mathrm{mm}^{-1}\right)$ & 33.97 \\
\hline Crystal size $(\mathrm{mm})$ & $0.10 \times 0.10 \times 0.10$ \\
\hline \multicolumn{2}{|l|}{ Data collection } \\
\hline Diffractometer & Bruker Kappa APEXII CCD \\
\hline Absorption correction & $\begin{array}{l}\text { Multi-scan ( } S A D A B S \text {; Bruker, } \\
\text { 2004) }\end{array}$ \\
\hline$T_{\min }, T_{\max }$ & $0.578,0.746$ \\
\hline $\begin{array}{l}\text { No. of measured, independent and } \\
\text { observed }[I>2 \sigma(I)] \text { reflections }\end{array}$ & $4109,489,387$ \\
\hline$R_{\text {int }}$ & 0.047 \\
\hline$(\sin \theta / \lambda)_{\max }\left(\AA^{-1}\right)$ & 0.718 \\
\hline \multicolumn{2}{|l|}{ Refinement } \\
\hline$R\left[F^{2}>2 \sigma\left(F^{2}\right)\right], w R\left(F^{2}\right), S$ & $0.047,0.113,1.42$ \\
\hline No. of reflections & 489 \\
\hline No. of parameters & 21 \\
\hline$\Delta \rho_{\max }, \Delta \rho_{\min }\left(\mathrm{e} \AA^{-3}\right)$ & $1.88,-2.11$ \\
\hline
\end{tabular}

Computer programs: APEX2 and SAINT (Bruker, 2004), SHELXT2014/4 (Sheldrick, 2015a), SHELXL2018/3 (Sheldrick, 2015b) and VESTA (Momma \& Izumi, 2011).

(Fig. 3). The thermal expansion is greater along the $a$ axis than the $c$ axis. Besides the anisotropy in the lattice parameters, the atomic positions did not vary significantly with temperature, and therefore the bond lengths change with temperature as dictated by the lattice parameters alone.

\section{Synthesis and crystallization}

Crystals of $\mathrm{TlMgCl}_{3}$ were grown from the melt using the vertical Bridgman method. High purity beads of $\mathrm{TlCl}$ and $\mathrm{MgCl}_{2}$ were combined in a stoichiometric ratio and sealed in a quartz ampoule under vacuum $\left(10^{-6}\right.$ Torr $)$. The crystal was grown with a translation speed of $0.5 \mathrm{~mm} \mathrm{~h}^{-1}$ and was cooled over $72 \mathrm{~h}$. To protect the moisture-sensitive reactants and products, all preparations before and after synthesis were conducted inside an argon-filled glove box.

\section{Refinement}

SC-XRD was conducted on a Bruker Kappa APEXII CCD diffractometer. The crystal was protected from moisture by oil during mounting and by an Oxford dry nitrogen gas cryostream system during data collection at $290 \mathrm{~K}$. Crystal data, data collection and structure refinement details are summarized in Table 2.

Powder high-temperature ND measurements were obtained using the high-pressure preferred orientation (HIPPO) neutron diffractometer at the short-pulsed spallation neutron source of the Lujan Neutron Scattering Center at Los Alamos National Laboratory (Wenk et al., 2003; Vogel et al., 2004). Powder samples were sealed under argon in vanadium tubes to protect from moisture during data collection. Time-of-flight data were collected with HIPPO detector panels of ${ }^{3} \mathrm{He}$ detector tubes arranged on five rings with nominal diffraction angles of $2 \theta=39,60,90,120$, and $144^{\circ}$. Count times were 90 minutes per dwell time. ND data were analyzed for all five rings simultaneously using the Rietveld method implemented in the GSAS code (Larson \& Von Dreele, 2004) and automated by scripts through gsaslanguage (Vogel, 2011). To yield reliable absolute lattice parameters, the DIFC instrument calibration parameters were fitted for the room-temperature data using the lattice parameters from SC-XRD and were kept constant for the rest of the ND data at higher temperatures. For more details on the data collection and refinement of these neutron diffraction data, see Onken et al. (2018).

The thermal expansion tensor was generated using a quadratic fit to the lattice parameters $\left(R^{2}=0.999\right)$, using the Thermal Expansion Visualization (TEV) program (Langreiter \& Kahlenberg, 2015).

\section{Acknowledgements}

The single-crystal structure determination was provided by the X-ray Analytical Facility at the University of California, Santa Barbara (Dr Guang Wu, Lab Manager).

\section{Funding information}

Funding for this research was provided by: U.S. Defense Threat Reduction Agency (DTRA) [contract No. HDTRA1931194 to Lawrence Berkeley National Laboratory (LBNL) authors]; U.S. Department of Energy, National Nuclear Security Administration (NNSA), Office of Defense Nuclear Nonproliferation (DNN) (contract No. AC02-05CH11231 to LBNL authors); U.S. Department of Energy, NNSA [contract No. 89233218NCA000001 to Los Alamos National Laboratory (LANL)].

\section{References}

Arai, M., Fujimoto, Y., Koshimizu, M., Yanagida, T. \& Asai, K. (2020). J. Alloy Compd. 823, 3-7.

Beznosikov, B. V. (1978). Sov. Phys. Crystallogr. (translated from Kristallografia), 23, 61-63.

Bruker (2004). APEX2, SAINT and SADABS. Bruker AXS Inc., Madison, Wisconsin, USA.

Derenzo, S. E. \& Choong, W.-S. (2009). IEEE Nucl. Sci. Sym. Conf. R. pp. $1-6$.

Devaney, K. O., Freedman, M. R., McPherson, G. L. \& Atwood, J. L. (1981). Inorg. Chem. 20, 140-145.

Fujimoto, Y., Koshimizu, M., Yanagida, T., Okada, G., Saeki, K. \& Asai, K. (2016). Jpn J. Appl. Phys. 55, 090301.

Goodyear, J., Steigmann, G. A. \& Ali, E. M. (1977). Acta Cryst. B33, 256-258.

Hawrami, R., Ariesanti, E., Wei, H., Finkelstein, J., Glodo, J. \& Shah, K. S. (2017). J. Cryst. Growth, 475, 216-219.

Langreiter, T. \& Kahlenberg, V. (2015). Crystals, 5, 143-153.

Larson, A. C. \& Von Dreele, R. B. (2004). GSAS. Report LAUR 86748. Los Alamos National Laboratory, New Mexico, USA.

McPherson, G. L., Kistenmacher, T. J. \& Stucky, G. D. (1970). J. Chem. Phys. 52, 815-824.

Momma, K. \& Izumi, F. (2011). J. Appl. Cryst. 44, 1272-1276. 
Onken, D. R., Williams, R. T., Perrodin, D., Shalapska, T., Bourret, E. D., Tremsin, A. S. \& Vogel, S. C. (2018). J. Appl. Cryst. 51, 498504.

Sheldrick, G. M. (2015a). Acta Cryst. A71, 3-8.

Sheldrick, G. M. (2015b). Acta Cryst. C71, 3-8.
Vogel, S. C. (2011). J. Appl. Cryst. 44, 873-877.

Vogel, S. C., Hartig, C., Lutterotti, L., Von Dreele, R. B., Wenk, H.-R. \& Williams, D. J. (2004). Powder Diffr. 19, 65-68.

Wenk, H.-R., Lutterotti, L. \& Vogel, S. (2003). Nucl. Instrum. Methods Phys. Res. A, 515, 575-588. 


\section{supporting information}

Acta Cryst. (2020). E76, 1716-1719 [https://doi.org/10.1107/S2056989020013201]

\section{The crystal structure of $\mathrm{TIMgCl}_{3}$ from $290 \mathrm{~K}$ to $725 \mathrm{~K}$}

\section{Drew R. Onken, Didier Perrodin, Sven C. Vogel, Edith D. Bourret and Federico Moretti}

\section{Computing details}

Data collection: APEX2 (Bruker, 2004); cell refinement: SAINT (Bruker, 2004); data reduction: SAINT (Bruker, 2004); program(s) used to solve structure: SHELXT2014/4 (Sheldrick, 2015a); program(s) used to refine structure:

SHELXL2018/3 (Sheldrick, 2015b); molecular graphics: VESTA (Momma \& Izumi, 2011); software used to prepare material for publication: SHELXL2018/3 (Sheldrick, 2015b).

Thallium magnesium trichloride

Crystal data

$\mathrm{TlMgCl}_{3}$

$M_{r}=335.03$

Hexagonal, $P 6_{3} / m m c$

$a=7.0228(4) \AA$

$c=17.4934(15) \AA$

$V=747.18(11) \AA^{3}$

$Z=6$

$F(000)=864$

Data collection

Bruker Kappa APEXII CCD diffractometer

$\omega$ scans

Absorption correction: multi-scan

(SADABS; Bruker, 2004)

$T_{\min }=0.578, T_{\max }=0.746$

4109 measured reflections

\section{Refinement}

Refinement on $F^{2}$

Least-squares matrix: full

$R\left[F^{2}>2 \sigma\left(F^{2}\right)\right]=0.047$

$w R\left(F^{2}\right)=0.113$

$S=1.42$

489 reflections

21 parameters

Special details
$D_{\mathrm{x}}=4.467 \mathrm{Mg} \mathrm{m}^{-3}$

Mo $K \alpha$ radiation, $\lambda=0.71073 \AA$

Cell parameters from 1053 reflections

$\theta=3.6-28.2^{\circ}$

$\mu=33.97 \mathrm{~mm}^{-1}$

$T=290 \mathrm{~K}$

Block, colorless

$0.10 \times 0.10 \times 0.10 \mathrm{~mm}$

489 independent reflections

387 reflections with $I>2 \sigma(I)$

$R_{\text {int }}=0.047$

$\theta_{\text {max }}=30.7^{\circ}, \theta_{\min }=2.3^{\circ}$

$h=-8 \rightarrow 9$

$k=-8 \rightarrow 8$

$l=-23 \rightarrow 25$

0 restraints

Primary atom site location: dual

$w=1 /\left[\sigma^{2}\left(F_{\mathrm{o}}^{2}\right)+23.1798 P\right]$

where $P=\left(F_{\mathrm{o}}^{2}+2 F_{\mathrm{c}}{ }^{2}\right) / 3$

$(\Delta / \sigma)_{\max }<0.001$

$\Delta \rho_{\max }=1.88 \mathrm{e} \AA^{-3}$

$\Delta \rho_{\min }=-2.11$ e $\AA^{-3}$

Geometry. All esds (except the esd in the dihedral angle between two 1.s. planes) are estimated using the full covariance matrix. The cell esds are taken into account individually in the estimation of esds in distances, angles and torsion angles; correlations between esds in cell parameters are only used when they are defined by crystal symmetry. An approximate (isotropic) treatment of cell esds is used for estimating esds involving l.s. planes. 
Fractional atomic coordinates and isotropic or equivalent isotropic displacement parameters $\left(\hat{A}^{2}\right)$

\begin{tabular}{lllll}
\hline & $x$ & $y$ & $z$ & $U_{\text {iso }} * / U_{\text {eq }}$ \\
\hline $\mathrm{T} 11$ & 0.000000 & 0.000000 & 0.250000 & $0.0375(5)$ \\
$\mathrm{T} 12$ & 0.333333 & 0.666667 & $0.09002(7)$ & $0.0395(4)$ \\
$\mathrm{Mg} 1$ & 0.666667 & 0.333333 & $0.3404(4)$ & $0.0115(14)$ \\
$\mathrm{Mg} 2$ & 1.000000 & 1.000000 & 0.500000 & $0.017(2)$ \\
$\mathrm{C} 11$ & $0.5075(4)$ & $0.0150(8)$ & 0.250000 & $0.0211(9)$ \\
$\mathrm{Cl} 2$ & $0.8336(4)$ & $0.6671(9)$ & $0.4185(2)$ & $0.0379(10)$ \\
\hline
\end{tabular}

Atomic displacement parameters $\left(\AA^{2}\right)$

\begin{tabular}{lllllll}
\hline & $U^{11}$ & $U^{22}$ & $U^{\beta 3}$ & $U^{12}$ & $U^{13}$ & $U^{23}$ \\
\hline $\mathrm{T} 11$ & $0.0378(7)$ & $0.0378(7)$ & $0.0369(8)$ & $0.0189(3)$ & 0.000 & 0.000 \\
$\mathrm{~T} 12$ & $0.0353(5)$ & $0.0353(5)$ & $0.0479(7)$ & $0.0176(2)$ & 0.000 & 0.000 \\
$\mathrm{Mg} 1$ & $0.011(2)$ & $0.011(2)$ & $0.013(3)$ & $0.0053(11)$ & 0.000 & 0.000 \\
$\mathrm{Mg} 2$ & $0.019(4)$ & $0.019(4)$ & $0.014(5)$ & $0.0094(19)$ & 0.000 & 0.000 \\
$\mathrm{C} 11$ & $0.0224(18)$ & $0.011(2)$ & $0.0261(19)$ & $0.0055(10)$ & 0.000 & 0.000 \\
$\mathrm{C} 12$ & $0.0418(18)$ & $0.027(2)$ & $0.0399(18)$ & $0.0135(10)$ & $-0.0123(9)$ & $-0.0246(18)$ \\
\hline
\end{tabular}

Geometric parameters $\left(\AA,{ }^{o}\right)$

\begin{tabular}{|c|c|c|c|}
\hline $\mathrm{T} 11-\mathrm{Cl1}^{\mathrm{i}}$ & $3.5126(2)$ & $\mathrm{Tl} 2-\mathrm{Cl}^{\mathrm{x}}$ & $3.5146(3)$ \\
\hline $\mathrm{T} 11-\mathrm{Cl1}^{\mathrm{ii}}$ & $3.5126(2)$ & $\mathrm{Tl} 2-\mathrm{Cl} 2^{\mathrm{xvi}}$ & $3.5146(3)$ \\
\hline $\mathrm{T} 11-\mathrm{C} 11^{\mathrm{iii}}$ & $3.5126(2)$ & $\mathrm{T} 12-\mathrm{Cl} 2^{\mathrm{xvii}}$ & $3.622(5)$ \\
\hline $\mathrm{T} 11-\mathrm{Cl1}$ & $3.5126(2)$ & $\mathrm{T} 12-\mathrm{C} 12^{\mathrm{xviii}}$ & $3.622(5)$ \\
\hline $\mathrm{T} 11-\mathrm{Cl1}^{\mathrm{iv}}$ & $3.5126(2)$ & $\mathrm{T} 12-\mathrm{Cl} 2^{\mathrm{xix}}$ & $3.622(5)$ \\
\hline $\mathrm{Tl1}-\mathrm{Cl1}^{\mathrm{v}}$ & $3.5126(2)$ & $\mathrm{Mg} 1-\mathrm{Cl} 2$ & $2.448(6)$ \\
\hline $\mathrm{T} 11-\mathrm{Cl} 2^{\mathrm{vi}}$ & $3.576(5)$ & $\mathrm{Mg} 1-\mathrm{Cl} 2^{\mathrm{ii}}$ & $2.448(6)$ \\
\hline $\mathrm{T} 11-\mathrm{Cl} 2^{\mathrm{vii}}$ & $3.576(5)$ & $\mathrm{Mg} 1-\mathrm{Cl} 2^{\mathrm{vii}}$ & $2.448(6)$ \\
\hline $\mathrm{Tl} 1-\mathrm{Cl} 2^{\text {viii }}$ & $3.576(5)$ & $\mathrm{Mg} 1-\mathrm{Cl1}^{\mathrm{vii}}$ & $2.499(6)$ \\
\hline $\mathrm{T} 11-\mathrm{Cl} 2^{\mathrm{ix}}$ & $3.576(5)$ & $\mathrm{Mg} 1-\mathrm{Cl1}$ & $2.499(6)$ \\
\hline $\mathrm{T} 11-\mathrm{Cl} 2^{\mathrm{x}}$ & $3.576(5)$ & $\mathrm{Mg} 1-\mathrm{Cl} 1^{\mathrm{ii}}$ & $2.499(6)$ \\
\hline $\mathrm{T} 11-\mathrm{Cl} 2^{\mathrm{xi}}$ & $3.576(5)$ & $\mathrm{Mg} 1-\mathrm{Mg} 1^{\mathrm{xvi}}$ & $3.162(13)$ \\
\hline $\mathrm{T} 12-\mathrm{C} 11^{\mathrm{ii}}$ & $3.510(3)$ & $\mathrm{Mg} 2-\mathrm{Cl}^{\mathrm{xx}}$ & $2.476(4)$ \\
\hline $\mathrm{T} 12-\mathrm{Cl1}{ }^{\mathrm{xii}}$ & $3.510(3)$ & $\mathrm{Mg} 2-\mathrm{Cl} 2^{\mathrm{xxi}}$ & $2.476(4)$ \\
\hline $\mathrm{Tl} 2-\mathrm{Cl1}^{\mathrm{iv}}$ & $3.510(3)$ & $\mathrm{Mg} 2-\mathrm{Cl} 2^{\mathrm{xxii}}$ & $2.476(4)$ \\
\hline $\mathrm{T} 12-\mathrm{Cl} 2^{\mathrm{xiii}}$ & $3.5146(3)$ & $\mathrm{Mg} 2-\mathrm{Cl} 2^{\text {xxiii }}$ & $2.476(4)$ \\
\hline $\mathrm{T} 12-\mathrm{Cl}^{\mathrm{xiv}}$ & $3.5146(3)$ & $\mathrm{Mg} 2-\mathrm{Cl} 2^{\text {xxiv }}$ & $2.476(4)$ \\
\hline $\mathrm{T} 12-\mathrm{Cl} 2^{\text {viii }}$ & $3.5146(3)$ & $\mathrm{Mg} 2-\mathrm{Cl} 2$ & $2.476(4)$ \\
\hline $\mathrm{T} 12-\mathrm{Cl}^{\mathrm{xv}}$ & $3.5146(3)$ & & \\
\hline $\mathrm{Cl} 11^{\mathrm{i}}-\mathrm{T} 11-\mathrm{C} 11^{\mathrm{ii}}$ & 120.0 & $\mathrm{Cl1} 1^{\mathrm{iv}}-\mathrm{T} 12-\mathrm{Cl} 2^{\mathrm{xvi}}$ & $123.81(8)$ \\
\hline $\mathrm{C} 11^{\mathrm{i}-}-\mathrm{T} 11-\mathrm{C} 11^{\mathrm{iii}}$ & $57.02(16)$ & $\mathrm{Cl} 2^{\mathrm{xiii}}-\mathrm{T} 12-\mathrm{Cl} 2^{\mathrm{xvi}}$ & $175.12(14)$ \\
\hline $\mathrm{C} 11^{\mathrm{ii}}-\mathrm{T} 11-\mathrm{C} 11^{\mathrm{iii}}$ & $177.02(16)$ & $\mathrm{Cl} 2^{\mathrm{xiv}}-\mathrm{T} 12-\mathrm{Cl} 2^{\mathrm{xvi}}$ & $119.820(11)$ \\
\hline $\mathrm{C} 11^{\mathrm{i}-}-\mathrm{T} 11-\mathrm{C} 11$ & $62.98(16)$ & $\mathrm{Cl} 2^{\mathrm{viii}}-\mathrm{T} 12-\mathrm{Cl} 2^{\mathrm{xvi}}$ & $119.820(10)$ \\
\hline $\mathrm{C} 11 \mathrm{ii}-\mathrm{T} 11-\mathrm{Cl} 1$ & $57.02(16)$ & $\mathrm{Cl} 2^{\mathrm{xv}}-\mathrm{Tl} 2-\mathrm{Cl}^{\mathrm{xvi}}$ & $59.85(17)$ \\
\hline $\mathrm{C} 11^{\mathrm{iii}}-\mathrm{T} 11-\mathrm{C} 11$ & 120.0 & $\mathrm{Cl} 2^{\mathrm{x}}-\mathrm{T} 12-\mathrm{Cl} 2^{\mathrm{xvi}}$ & $60.03(17)$ \\
\hline
\end{tabular}




$$
\begin{aligned}
& \mathrm{C} 11^{\mathrm{i}}-\mathrm{Tl} 1-\mathrm{Cl1}^{\mathrm{iv}} \\
& \mathrm{C} 11^{\mathrm{ii}-\mathrm{T}} 11-\mathrm{C} 11^{\mathrm{iv}} \\
& \text { Cl1 } 1 \text { iii-Tl1- } \mathrm{Cl1}^{\mathrm{iv}} \\
& \mathrm{Cl} 1-\mathrm{Tl}-\mathrm{Cl1}^{\mathrm{iv}} \\
& \mathrm{C} 11^{\mathrm{i}}-\mathrm{T} 11-\mathrm{Cl1}^{\mathrm{v}} \\
& \mathrm{Cl} 1^{\mathrm{ii}}-\mathrm{T} 11-\mathrm{Cl}^{\mathrm{v}} \\
& \mathrm{Cl1} 1^{\mathrm{ii}}-\mathrm{Tl}-\mathrm{Cl1}^{\mathrm{v}} \\
& \mathrm{C} 11-\mathrm{T} 11-\mathrm{Cl1}^{\mathrm{v}} \\
& \text { Cl1 }{ }^{\mathrm{iv}}-\mathrm{Tl} 1-\mathrm{Cl1}^{\mathrm{v}} \\
& \mathrm{C} 11^{\mathrm{i}}-\mathrm{T} 11-\mathrm{Cl}^{\mathrm{vi}} \\
& \mathrm{C} 11^{\mathrm{ii}}-\mathrm{T} 11-\mathrm{Cl}^{\mathrm{vi}} \\
& \mathrm{C} 11^{\mathrm{iii}}-\mathrm{T} 11-\mathrm{Cl}^{2 \mathrm{vi}} \\
& \mathrm{Cl} 1-\mathrm{Tl} 1-\mathrm{Cl}^{\mathrm{vi}} \\
& \mathrm{Cl1} 1^{\mathrm{iv}}-\mathrm{T} 11-\mathrm{Cl}^{\mathrm{vi}} \\
& \mathrm{Cl1}{ }^{\mathrm{v}}-\mathrm{T} 11-\mathrm{Cl}^{\mathrm{vi}} \\
& \mathrm{Cl1}{ }^{\mathrm{i}}-\mathrm{Tl} 1-\mathrm{Cl}^{\mathrm{vii}} \\
& \mathrm{C} 11^{1 i}-\mathrm{T} 11-\mathrm{Cl} 2^{\text {vii }} \\
& \mathrm{C} 11^{\mathrm{iii}}-\mathrm{T} 11-\mathrm{Cl} 2^{\mathrm{vii}} \\
& \text { Cl1-Tl1-Cl2 }{ }^{\text {vii }} \\
& \mathrm{Cl1}{ }^{\text {iv }}-\mathrm{T} 11-\mathrm{Cl} 2^{\text {vii }} \\
& \mathrm{Cl1}{ }^{\mathrm{v}}-\mathrm{T} 11-\mathrm{Cl}^{\mathrm{vii}} \\
& \mathrm{Cl} 2^{\mathrm{vi}}-\mathrm{T} 11-\mathrm{Cl}^{\mathrm{vii}} \\
& \mathrm{Cl1}{ }^{i}-\mathrm{T} 11-\mathrm{Cl} 2^{\mathrm{viii}} \\
& \mathrm{C} 11^{1 i}-\mathrm{Tl} 1-\mathrm{Cl} 2^{\text {viii }}
\end{aligned}
$$

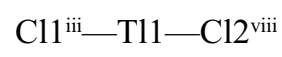

$$
\begin{aligned}
& \text { Cl1-Tl1-Cl2 }{ }^{\text {viii }} \\
& \mathrm{C} 11^{\mathrm{iv}}-\mathrm{T} 11-\mathrm{Cl} 2^{\text {viii }} \\
& \mathrm{Cl} 1^{\mathrm{v}}-\mathrm{Tl} 1-\mathrm{Cl}^{\text {viii }}
\end{aligned}
$$

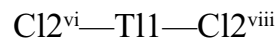

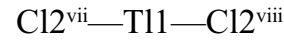

$$
\begin{aligned}
& \text { Cl1 }-\mathrm{T} 11-\mathrm{Cl} 2^{\mathrm{ix}} \\
& \mathrm{Cl1} 1^{\mathrm{ii}}-\mathrm{T} 11-\mathrm{Cl} 2^{\mathrm{ix}} \\
& \mathrm{C} 11^{\mathrm{iii}}-\mathrm{T} 11-\mathrm{Cl} 2^{\mathrm{ix}} \\
& \mathrm{Cl} 1-\mathrm{Tl} 1-\mathrm{Cl} 2^{\mathrm{ix}} \\
& \mathrm{Cl1}{ }^{\mathrm{iv}}-\mathrm{T} 11-\mathrm{Cl} 2^{\mathrm{ix}} \\
& \mathrm{C} 11^{\mathrm{v}}-\mathrm{T} 11-\mathrm{C} 12^{\mathrm{ix}} \\
& \mathrm{Cl} 2^{\mathrm{vi}}-\mathrm{T} 11-\mathrm{Cl} 2^{\mathrm{ix}}
\end{aligned}
$$

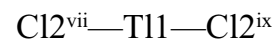

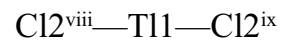

$$
\begin{aligned}
& \mathrm{C} 11^{\mathrm{i}}-\mathrm{T} 11-\mathrm{Cl} 2^{\mathrm{x}} \\
& \mathrm{C} 11^{\mathrm{ii}}-\mathrm{T} 11-\mathrm{Cl} 2^{\mathrm{x}}
\end{aligned}
$$

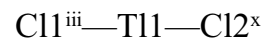

$$
\begin{aligned}
& \mathrm{Cl} 1-\mathrm{T} 11-\mathrm{Cl}^{\mathrm{x}} \\
& \mathrm{C} 11^{\mathrm{iv}}-\mathrm{T} 11-\mathrm{Cl} 2^{\mathrm{x}} \\
& \mathrm{C} 11^{\mathrm{v}}-\mathrm{T} 11-\mathrm{Cl}^{\mathrm{x}} \\
& \mathrm{Cl} 2^{\mathrm{vi}}-\mathrm{T} 11-\mathrm{Cl}^{\mathrm{x}} \\
& \mathrm{Cl} 2^{\text {vii }-\mathrm{T} 11-\mathrm{Cl}^{\mathrm{x}}} \\
& \mathrm{Cl} 2^{\text {viii- }} \mathrm{Tl} 1-\mathrm{Cl}^{\mathrm{x}}
\end{aligned}
$$

$177.02(16)$
$62.98(16)$
120.0
$120.000(1)$
$120.000(1)$
$119.999(1)$
$62.98(16)$
$177.02(16)$
$57.02(16)$
$60.17(6)$
$118.86(6)$
$60.17(6)$
$90.84(4)$
$118.86(6)$
$90.84(5)$
$90.84(4)$
$60.17(6)$
$118.86(6)$
$60.17(6)$
$90.84(4)$
$118.86(6)$
$147.12(6)$
$118.86(6)$
$90.84(5)$
$90.84(5)$
$118.86(6)$
$60.17(6)$
$60.17(6)$
$58.71(11)$
$147.12(6)$
$118.86(6)$
$90.84(5)$
$90.84(5)$
$118.86(6)$
$60.17(6)$
$60.17(6)$
$147.12(6)$
$58.71(11)$
$111.04(14)$
$90.84(4)$
$60.17(6)$
$118.86(6)$
$60.17(6)$
$90.84(5)$
$118.86(6)$
$58.71(11)$
$111.04(14)$
$58.71(11)$

$\mathrm{Cl1}{ }^{\mathrm{ii}}-\mathrm{T} 12-\mathrm{Cl} 2^{\mathrm{xvi}}$

$\mathrm{Cl1} 1^{\mathrm{xii}}-\mathrm{T} 12-\mathrm{Cl} 2^{\mathrm{xvii}}$

$\mathrm{Cl1} 1^{\mathrm{iv}}-\mathrm{T} 12-\mathrm{Cl}^{\mathrm{xvii}}$

$\mathrm{Cl} 2^{\mathrm{xiii}}-\mathrm{T} 12-\mathrm{Cl} 2^{\mathrm{xvii}}$

$\mathrm{Cl} 2^{\text {xiv }}-\mathrm{T} 12-\mathrm{Cl} 2^{\text {xvii }}$

$\mathrm{Cl}^{\text {viii }}-\mathrm{T} 12-\mathrm{Cl}^{\text {xvii }}$

$\mathrm{Cl} 2^{\mathrm{xv}}-\mathrm{T} 12-\mathrm{Cl} 2^{\mathrm{xvii}}$

$\mathrm{Cl} 2^{\mathrm{x}}-\mathrm{T} 12-\mathrm{Cl} 2^{\mathrm{xvii}}$

$\mathrm{Cl} 2^{\mathrm{xvi}}-\mathrm{T} 12-\mathrm{Cl}^{\mathrm{xvii}}$

$\mathrm{C} 11^{\mathrm{ii}}-\mathrm{T} 12-\mathrm{C} 12^{\mathrm{xvii}}$

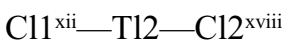

$\mathrm{C} 11^{\text {iv }}-\mathrm{T} 12-\mathrm{Cl} 2^{\text {xvii }}$

$\mathrm{Cl} 2^{\text {xiii }}-\mathrm{T} 12-\mathrm{Cl} 2^{\text {xviii }}$

$\mathrm{Cl} 2^{\mathrm{xiv}}-\mathrm{T} 12-\mathrm{Cl} 2^{\mathrm{xviii}}$

$\mathrm{Cl} 2^{\text {viii- }}-\mathrm{Tl} 2-\mathrm{Cl}^{\text {xviii }}$

$\mathrm{Cl} 2^{\mathrm{xv}}-\mathrm{T} 12-\mathrm{Cl} 2^{\mathrm{xvii}}$

$\mathrm{Cl} 2^{\mathrm{x}}-\mathrm{T} 12-\mathrm{Cl}^{\mathrm{xviii}}$

$\mathrm{Cl} 2^{\mathrm{xvi}}-\mathrm{T} 12-\mathrm{Cl} 2^{\mathrm{xvii}}$

$\mathrm{Cl} 2^{\mathrm{xvii}}-\mathrm{T} 12-\mathrm{Cl} 2^{\mathrm{xvii}}$

$\mathrm{Cl} 1^{\mathrm{ii}}-\mathrm{T} 12-\mathrm{Cl} 2^{\mathrm{xix}}$

$\mathrm{Cl1}{ }^{\mathrm{xii}}-\mathrm{T} 12-\mathrm{Cl} 2^{\mathrm{xix}}$

$\mathrm{Cl} 11^{\mathrm{iv}}-\mathrm{T} 12-\mathrm{Cl}^{\mathrm{xix}}$

$\mathrm{Cl} 2^{\mathrm{xiii}}-\mathrm{T} 12-\mathrm{Cl} 2^{\mathrm{xix}}$

$\mathrm{Cl} 2^{\text {xiv }}-\mathrm{T} 12-\mathrm{Cl}^{\text {xix }}$

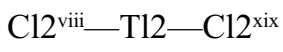

$\mathrm{Cl} 2^{\mathrm{xv}}-\mathrm{T} 12-\mathrm{Cl} 2^{\mathrm{xix}}$

$\mathrm{Cl} 2^{x}-\mathrm{T} 12-\mathrm{Cl}^{\mathrm{xix}}$

$\mathrm{Cl} 2^{\mathrm{xvi}}-\mathrm{T} 12-\mathrm{Cl} 2^{\mathrm{xix}}$

$\mathrm{Cl} 2^{\text {xvi }}-\mathrm{T} 12-\mathrm{Cl}^{\mathrm{xix}}$

$\mathrm{Cl} 2^{\text {xiii }-}-\mathrm{Tl} 2-\mathrm{Cl}_{2}{ }^{\mathrm{xix}}$

$\mathrm{Cl} 2-\mathrm{Mg} 1-\mathrm{Cl} 2^{\mathrm{ii}}$

$\mathrm{Cl} 2-\mathrm{Mg} 1-\mathrm{Cl}^{\text {vii }}$

$\mathrm{Cl} 2^{\mathrm{ii}}-\mathrm{Mg} 1-\mathrm{Cl}^{\mathrm{vii}}$

$\mathrm{Cl} 2-\mathrm{Mg} 1-\mathrm{Cl1}^{\text {vii }}$

$\mathrm{Cl} 2^{\mathrm{ii}}-\mathrm{Mg} 1-\mathrm{Cl} 1^{\mathrm{vii}}$

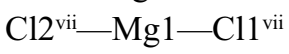

$\mathrm{Cl} 2-\mathrm{Mg} 1-\mathrm{Cl} 1$

$\mathrm{Cl} 2^{\mathrm{ii}}-\mathrm{Mg} 1-\mathrm{Cl} 1$

$\mathrm{Cl}{ }^{\text {vii }}-\mathrm{Mg} 1-\mathrm{Cl} 1$

$\mathrm{Cl1}$ vii-Mg1-Cl1

$\mathrm{Cl} 2-\mathrm{Mg} 1-\mathrm{Cl}^{\mathrm{ii}}$

$\mathrm{Cl} 2^{\mathrm{ii}}-\mathrm{Mg} 1-\mathrm{Cl}^{\mathrm{ii}}$

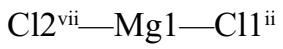

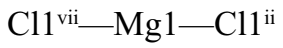

$\mathrm{C} 11-\mathrm{Mg} 1-\mathrm{Cl}^{\mathrm{ii}}$

$\mathrm{Cl} 2^{\mathrm{xx}}-\mathrm{Mg} 2-\mathrm{Cl}^{\mathrm{xxi}}$

$\mathrm{Cl} 2^{\mathrm{xx}}-\mathrm{Mg} 2-\mathrm{Cl}^{\text {xxii }}$

$\mathrm{Cl} 2^{\mathrm{xxi}}-\mathrm{Mg} 2-\mathrm{Cl}^{\mathrm{xxii}}$
$176.96(10)$

$119.42(7)$

$119.42(7)$

58.64 (11)

$58.64(11)$

$88.00(9)$

88.00 (9)

$116.71(6)$

$116.71(6)$

$119.42(7)$

$176.96(10)$

$119.42(7)$

88.00 (9)

$116.71(6)$

$58.64(11)$

116.71 (6)

58.64 (11)

$88.00(9)$

58.07 (11)

$119.42(7)$

$119.42(8)$

$176.96(10)$

$116.71(6)$

88.00 (9)

$116.71(6)$

58.64 (11)

88.00 (9)

58.64 (11)

58.07 (11)

58.07 (11)

$91.8(2)$

$91.8(2)$

$91.8(2)$

$91.84(10)$

$91.84(10)$

$174.7(3)$

174.7 (3)

$91.84(10)$

91.84 (10)

84.3 (2)

91.84 (10)

174.7 (3)

91.84 (10)

84.3 (2)

84.3 (2)

90.17 (16)

89.83 (16)

180.0 


\begin{tabular}{|c|c|}
\hline $\mathrm{C} 12^{\mathrm{ix}}-\mathrm{T} 11-\mathrm{C} 12^{\mathrm{x}}$ & $147.12(6)$ \\
\hline $\mathrm{Cl1} 1^{\mathrm{i}}-\mathrm{T} 11-\mathrm{Cl} 2^{\mathrm{xi}}$ & $60.17(6)$ \\
\hline $\mathrm{Cl} 11^{\mathrm{ii}}-\mathrm{Tl} 1-\mathrm{Cl} 2^{\mathrm{xi}}$ & $118.86(6)$ \\
\hline 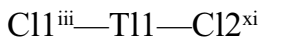 & $60.17(6)$ \\
\hline $\mathrm{Cl} 1-\mathrm{T} 11-\mathrm{Cl} 2^{\mathrm{xi}}$ & $90.84(4)$ \\
\hline $\mathrm{Cl} 1^{\mathrm{iv}}-\mathrm{T} 11-\mathrm{Cl}^{\mathrm{xi}}$ & $118.86(6)$ \\
\hline $\mathrm{Cl} 1^{\mathrm{v}}-\mathrm{T} 11-\mathrm{Cl} 2^{\mathrm{xi}}$ & $90.84(5)$ \\
\hline $\mathrm{Cl} 2^{\mathrm{vi}}-\mathrm{T} 11-\mathrm{Cl}^{\mathrm{xi}}$ & $111.04(14)$ \\
\hline $\mathrm{Cl} 2^{\mathrm{vii}}-\mathrm{Tl} 1-\mathrm{Cl}^{\mathrm{xi}}$ & $58.71(11)$ \\
\hline $\mathrm{Cl} 2^{\mathrm{viii}}-\mathrm{T} 11-\mathrm{Cl} 2^{\mathrm{xi}}$ & $147.12(6)$ \\
\hline $\mathrm{Cl} 2^{\mathrm{ix}}-\mathrm{T} 11-\mathrm{Cl} 2^{\mathrm{xi}}$ & $58.71(11)$ \\
\hline $\mathrm{Cl} 2^{\mathrm{x}}-\mathrm{T} 11-\mathrm{Cl} 2^{\mathrm{xi}}$ & $147.12(6)$ \\
\hline $\mathrm{C} 11^{\mathrm{ii}}-\mathrm{T} 12-\mathrm{C} 11^{\mathrm{xii}}$ & $63.03(10)$ \\
\hline 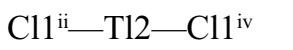 & $63.03(10)$ \\
\hline 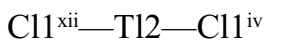 & $63.03(10)$ \\
\hline $\mathrm{C} 11^{\mathrm{ii}}-\mathrm{T} 12-\mathrm{Cl} 2^{\mathrm{xiii}}$ & $123.81(8)$ \\
\hline $\mathrm{C} 11^{\mathrm{xii}}-\mathrm{T} 12-\mathrm{Cl} 2^{\mathrm{xiii}}$ & $91.92(9)$ \\
\hline $\mathrm{C} 11^{\mathrm{iv}}-\mathrm{T} 12-\mathrm{Cl} 2^{\mathrm{xiii}}$ & $60.79(8)$ \\
\hline $\mathrm{Cl} 1^{\mathrm{ii}}-\mathrm{T} 12-\mathrm{Cl} 2^{\mathrm{xiv}}$ & $123.81(8)$ \\
\hline $\mathrm{C} 11^{\mathrm{xii}}-\mathrm{T} 12-\mathrm{Cl} 2^{\mathrm{xiv}}$ & $60.79(8)$ \\
\hline $\mathrm{C} 11^{\mathrm{iv}}-\mathrm{T} 12-\mathrm{Cl} 2^{\mathrm{xiv}}$ & $91.92(9)$ \\
\hline $\mathrm{Cl} 2^{\mathrm{xiii}}-\mathrm{T} 12-\mathrm{Cl} 2^{\mathrm{xiv}}$ & $59.85(17)$ \\
\hline $\mathrm{Cl} 1^{\mathrm{ii}}-\mathrm{T} 12-\mathrm{Cl} 2^{\mathrm{viii}}$ & $91.92(9)$ \\
\hline 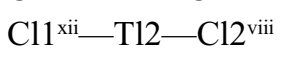 & $123.81(8)$ \\
\hline $\mathrm{C} 11^{\mathrm{iv}}-\mathrm{T} 12-\mathrm{Cl} 2^{\mathrm{viii}}$ & $60.79(8)$ \\
\hline $\mathrm{Cl} 2^{\mathrm{xiii}}-\mathrm{T} 12-\mathrm{Cl} 2^{\mathrm{viii}}$ & $60.03(17)$ \\
\hline $\mathrm{Cl} 2^{\mathrm{xiv}}-\mathrm{Tl} 2-\mathrm{Cl} 2^{\mathrm{viii}}$ & $119.820(10)$ \\
\hline $\mathrm{Cl} 1^{\mathrm{ii}}-\mathrm{T} 12-\mathrm{Cl} 2^{\mathrm{xv}}$ & $91.92(9)$ \\
\hline $\mathrm{Cl} 1^{\mathrm{xii}}-\mathrm{T} 12-\mathrm{Cl} 2^{\mathrm{xv}}$ & $60.79(8)$ \\
\hline $\mathrm{Cl} 1^{\mathrm{iv}}-\mathrm{T} 12-\mathrm{Cl} 2^{\mathrm{xv}}$ & $123.81(8)$ \\
\hline $\mathrm{Cl} 2^{\mathrm{xiii}}-\mathrm{T} 12-\mathrm{Cl} 2^{\mathrm{xv}}$ & $119.820(10)$ \\
\hline $\mathrm{Cl} 2^{\mathrm{xiv}}-\mathrm{T} 12-\mathrm{Cl} 2^{\mathrm{xv}}$ & $60.03(17)$ \\
\hline $\mathrm{Cl} 2^{\mathrm{viii}}-\mathrm{T} 12-\mathrm{Cl} 2^{\mathrm{xv}}$ & $175.12(14)$ \\
\hline 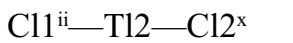 & $60.79(8)$ \\
\hline $\mathrm{C} 11^{\mathrm{xii}}-\mathrm{T} 12-\mathrm{Cl} 2^{\mathrm{x}}$ & $123.81(8)$ \\
\hline $\mathrm{C} 11^{\mathrm{iv}}-\mathrm{T} 12-\mathrm{Cl} 2^{\mathrm{x}}$ & $91.92(9)$ \\
\hline $\mathrm{Cl} 2^{\mathrm{xiii}}-\mathrm{T} 12-\mathrm{Cl} 2^{\mathrm{x}}$ & $119.820(11)$ \\
\hline $\mathrm{Cl} 2^{\mathrm{xiv}}-\mathrm{T} 12-\mathrm{C} 12^{\mathrm{x}}$ & $175.12(14)$ \\
\hline 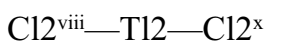 & $59.85(17)$ \\
\hline $\mathrm{Cl} 2^{\mathrm{xv}}-\mathrm{T} 12-\mathrm{Cl} 2^{\mathrm{x}}$ & $119.820(11)$ \\
\hline $\mathrm{C} 11^{\mathrm{ii}}-\mathrm{T} 12-\mathrm{Cl} 2^{\mathrm{xvi}}$ & $60.79(8)$ \\
\hline $\mathrm{C} 11^{\mathrm{xii}}-\mathrm{T} 12-\mathrm{C} 12^{\mathrm{xvi}}$ & $91.92(9)$ \\
\hline
\end{tabular}

\begin{tabular}{|c|c|}
\hline $\mathrm{Cl} 2^{\mathrm{xx}}-\mathrm{Mg} 2-\mathrm{Cl} 2^{\mathrm{xxiii}}$ & $90.17(16)$ \\
\hline $\mathrm{Cl} 2^{\mathrm{xxi}}-\mathrm{Mg} 2-\mathrm{Cl} 2^{\mathrm{xxiii}}$ & $90.17(17)$ \\
\hline $\mathrm{Cl} 2^{\mathrm{xxi}}-\mathrm{Mg} 2-\mathrm{Cl} 2^{\mathrm{xxiii}}$ & 89.83 (17) \\
\hline $\mathrm{Cl} 2^{\mathrm{xx}}-\mathrm{Mg} 2-\mathrm{Cl} 2^{\mathrm{xxiv}}$ & $89.83(16)$ \\
\hline $\mathrm{Cl} 2^{\mathrm{xxi}}-\mathrm{Mg} 2-\mathrm{Cl} 2^{\mathrm{xxiv}}$ & $89.83(17)$ \\
\hline $\mathrm{Cl} 2^{\mathrm{xxi}}-\mathrm{Mg} 2-\mathrm{Cl} 2^{\mathrm{xxiv}}$ & 90.17 (17) \\
\hline $\mathrm{Cl} 2^{\mathrm{xxii}}-\mathrm{Mg} 2-\mathrm{Cl} 2^{\mathrm{xxiv}}$ & 180.0 \\
\hline $\mathrm{Cl} 2^{\mathrm{xx}}-\mathrm{Mg} 2-\mathrm{Cl} 2$ & 180.0 \\
\hline $\mathrm{Cl} 2^{\mathrm{xxi}}-\mathrm{Mg} 2-\mathrm{Cl} 2$ & $89.83(17)$ \\
\hline $\mathrm{Cl} 22^{\mathrm{xxi}}-\mathrm{Mg} 2-\mathrm{Cl} 2$ & 90.17 (17) \\
\hline $\mathrm{Cl} 2^{\mathrm{xxiii}}-\mathrm{Mg} 2-\mathrm{Cl} 2$ & $89.83(16)$ \\
\hline $\mathrm{Cl} 2^{\mathrm{xxiv}}-\mathrm{Mg} 2-\mathrm{Cl} 2$ & $90.17(16)$ \\
\hline 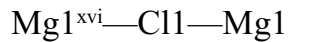 & $78.5(3)$ \\
\hline $\mathrm{Mg} 1^{\mathrm{xvi}}-\mathrm{C} 11-\mathrm{Tl} 2^{\mathrm{xxv}}$ & $87.89(12)$ \\
\hline $\mathrm{Mg} 1-\mathrm{Cl1}-\mathrm{T} 12^{\mathrm{xxv}}$ & $166.36(18)$ \\
\hline $\mathrm{Mg} 1^{\mathrm{xvi}}-\mathrm{Cl} 1-\mathrm{T} 12^{\mathrm{xxvi}}$ & $166.36(18)$ \\
\hline $\mathrm{Mg} 1-\mathrm{Cl} 1-\mathrm{T} 12^{\mathrm{xxvi}}$ & $87.89(12)$ \\
\hline $\mathrm{T} 12^{\mathrm{xxv}}-\mathrm{C} 11-\mathrm{T} 12^{\mathrm{xxvi}}$ & $105.74(13)$ \\
\hline $\mathrm{Mg} 1^{\mathrm{xvi}}-\mathrm{C} 11-\mathrm{T} 11^{\mathrm{xxvii}}$ & $91.16(6)$ \\
\hline $\mathrm{Mg} 1-\mathrm{Cl} 1-\mathrm{T} 11^{\mathrm{xxvii}}$ & $91.15(6)$ \\
\hline $\mathrm{T} 12^{\mathrm{xxy}}-\mathrm{C} 11-\mathrm{T} 11^{\mathrm{xxvii}}$ & $89.10(5)$ \\
\hline $\mathrm{T} 12^{\mathrm{xxvi}}-\mathrm{C} 11-\mathrm{T} 11^{\mathrm{xxvii}}$ & $89.10(5)$ \\
\hline $\mathrm{Mg} 1^{\mathrm{xvi}}-\mathrm{C} 11-\mathrm{T} 11$ & $91.15(6)$ \\
\hline $\mathrm{Mg} 1-\mathrm{C} 11-\mathrm{T} 11$ & $91.15(6)$ \\
\hline $\mathrm{T} 12^{\mathrm{xxv}}-\mathrm{C} 11-\mathrm{T} 11$ & $89.10(5)$ \\
\hline $\mathrm{T} 12^{\mathrm{xxv}}-\mathrm{C} 11-\mathrm{T} 11$ & $89.10(5)$ \\
\hline 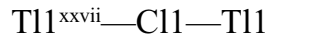 & $177.02(16)$ \\
\hline $\mathrm{Mg} 1-\mathrm{Cl} 2-\mathrm{Mg} 2$ & $178.8(3)$ \\
\hline $\mathrm{Mg} 1-\mathrm{Cl} 2-\mathrm{T} 12^{\mathrm{xvi}}$ & $88.60(7)$ \\
\hline $\mathrm{Mg} 2-\mathrm{Cl} 2-\mathrm{T} 12^{\mathrm{xvi}}$ & $91.44(7)$ \\
\hline $\mathrm{Mg} 1-\mathrm{Cl} 2-\mathrm{T} 12^{\mathrm{xxviii}}$ & $88.60(7)$ \\
\hline $\mathrm{Mg} 2-\mathrm{Cl} 2-\mathrm{T} 12^{\mathrm{x} x v i i i}$ & $91.44(7)$ \\
\hline $\mathrm{T} 12^{\mathrm{xvi}}-\mathrm{C} 12-\mathrm{T} 12^{\mathrm{xxviii}}$ & $175.12(14)$ \\
\hline $\mathrm{Mg} 1-\mathrm{Cl} 2-\mathrm{T} 11^{\text {xix }}$ & $90.52(17)$ \\
\hline $\mathrm{Mg} 2-\mathrm{Cl} 2-\mathrm{T} 11^{\mathrm{xxix}}$ & $90.67(16)$ \\
\hline $\mathrm{Tl} 2^{\mathrm{xvi}}-\mathrm{Cl} 2-\mathrm{T} 11^{\mathrm{xxix}}$ & $88.02(8)$ \\
\hline $\mathrm{T} 12^{\mathrm{xxviii}}-\mathrm{Cl} 2-\mathrm{T} 11^{\mathrm{xxix}}$ & $88.02(8)$ \\
\hline $\mathrm{Mg} 1-\mathrm{Cl} 2-\mathrm{T} 12^{\mathrm{xxx}}$ & $89.9(2)$ \\
\hline $\mathrm{Mg} 2-\mathrm{Cl} 2-\mathrm{T} 12^{\mathrm{xxx}}$ & $88.94(11)$ \\
\hline $\mathrm{T} 12^{\mathrm{xvi}}-\mathrm{C} 12-\mathrm{T} 12^{\mathrm{xxx}}$ & $91.99(9)$ \\
\hline $\mathrm{T} 12^{\mathrm{xxviii}}-\mathrm{Cl} 2-\mathrm{T} 12^{\mathrm{xxx}}$ & $91.99(9)$ \\
\hline $\mathrm{T} 11^{\mathrm{xxix}}-\mathrm{Cl} 2-\mathrm{T} 12^{\mathrm{xxx}}$ & $179.61(13)$ \\
\hline
\end{tabular}

Symmetry codes: (i) $-y, x-y-1, z$; (ii) $-x+y+1,-x+1, z$; (iii) $-x+y,-x, z$; (iv) $-y, x-y, z$; (v) $x-1, y, z$; (vi) $x-1, y-1,-z+1 / 2$; (vii) $-y+1, x-y, z$; (viii) $-x+y$, $-x+1,-z+1 / 2$; (ix) $-x+y,-x+1, z$; (x) $-y+1, x-y,-z+1 / 2$; (xi) $x-1, y-1, z$; (xii) $x, y+1, z$; (xiii) $x-1, y,-z+1 / 2$; (xiv) $-y+1, x-y+1,-z+1 / 2$; (xv) $-x+y+1$, $-x+2,-z+1 / 2$; (xvi) $x, y,-z+1 / 2$; (xvii) $x-y, x, z-1 / 2$; (xviii) $-x+1,-y+1, z-1 / 2$; (xix) $y,-x+y+1, z-1 / 2$; (xx) $-x+2,-y+2,-z+1$; (xxi) $x-y+1, x,-z+1$; (xxii) $-x+y+1,-x+2, z$; (xxiii) $y,-x+y+1,-z+1$; (xxiv) $-y+2, x-y+1, z$; (xxv) $x, y-1, z$; (xxvi) $x, y-1,-z+1 / 2$; (xxvii) $x+1, y, z$; (xxviii) $x+1, y,-z+1 / 2$; (xxix) $x+1, y+1, z$; (xxx) $-x+1,-y+1, z+1 / 2$. 